\section{Check for updates}

Cite this: Food Funct., 2022, 13, 1617

\title{
Effect of high-amylose starch branching enzyme II wheat mutants on starch digestibility in bread, product quality, postprandial satiety and glycaemic response $\uparrow$
}

\author{
Marina Corrado, (D) a Jennifer H. Ahn-Jarvis, ${ }^{a}$ Brendan Fahy, ${ }^{b}$ George M. Savva, ${ }^{a}$ \\ Cathrina $\mathrm{H}$. Edwards (D) * $\neq^{\mathrm{a}}$ and Brittany A. Hazard (D) $\ddagger^{\mathrm{a}, \mathrm{b}}$
}

High-amylose starch branching enzyme // (sbell) mutant wheat has potential to be low-glycaemic compared to conventional wheat; however, the effects of bread made from sbell wheat flour on glycaemic response and product quality require investigation. We report the impact of white bread made from sbell wheat flour on in vitro starch digestibility and product quality, and on postprandial glycaemia in vivo, compared to an isoglucidic wild-type (WT) control white bread. Starch in sbell bread was $20 \%$ less susceptible to in vitro amylolysis leading to $15 \%$ lower glycaemic response measured in vivo, compared to the WT control bread, without major effects on bread appearance or texture, measured instrumentally. Despite the early termination of the in vivo intervention study due to the COVID-19 outbreak ( $n=8$ out of 19), results from this study indicate that sbell wheat produces bread with lower starch digestibility than conventional white bread.

Received 14th September 2021 Accepted 6th December 2021 DOI: $10.1039 / \mathrm{d} 1 \mathrm{fo} 03085 \mathrm{j}$ rsc.li/food-function opportunities to develop slowly digested carbohydrate staple foods with lower glycaemic potency.

Starch structure can be manipulated in planta using Targeting Induced Local Lesions in Genomes (TILLING) technology ${ }^{8}$ to induce mutations in genes involved in starch biosynthesis. ${ }^{9}$ Wheat carrying mutations in starch branching enzyme II (sbeII) genes have been generated using TILLING, yielding starch with a higher proportion of amylose and increased resistance to amylase digestion, compared to conventional wheat starch. ${ }^{10,11}$ This allows the production of highamylose starch-based food products without the need of postextraction processing or supplementation with starches from other botanical sources, which was shown to be a suitable approach to lower glycaemic response to high glycaemic foods, such as bread. ${ }^{12,13}$

Previously, a sbeII mutant wheat was used to produce a semolina pudding with lower starch digestibility than the control pudding made from WT wheat. When tested in humans, however, the Glycaemic Index of the sbeII pudding was not significantly different from the WT control. ${ }^{14}$ Recently, two studies have investigated glycaemic responses to pasta and bread made from sbeII mutant wheat. Sissons et al. (2020), ${ }^{15}$ reported that a serving of pasta (spaghetti) made from a sbeIIa durum wheat mutant with starch made of $\sim 58 \%$ amylose, corresponding to $\sim 7 \% \mathrm{RS}$, led to a lower starch susceptibility to amylase digestion in vitro and lower glycaemic index measured in vivo, compared to the WT control. Belobrajdic

\footnotetext{
${ }^{a}$ Food Innovation and Health, Quadram Institute Bioscience, Norwich Research Park, UK. E-mail: cathrina.edwards@quadram.ac.uk

${ }^{b}$ Designing Future Wheat and Molecules from Nature, John Innes Centre, Norwich Research Park, UK

$\dagger$ Electronic supplementary information (ESI) available. See DOI: 10.1039/ d1fo03085j

$\$$ These authors contributed equally.
} 
et al. (2019), ${ }^{16}$ reported that bread made from high-amylose wheat flour lowered the postprandial glycaemic response by $39 \%$ and the insulinemic response by $24 \%$, however the bread provided $\sim 24 \mathrm{~g}$ less starch per $100 \mathrm{~g}$ and $\sim 7.5 \%$ less energy (kJ per $100 \mathrm{~g}$ ) than the control bread. Therefore, it is not clear if the attenuated postprandial glycaemic response following high-amylose bread in the Belobrajdic et al. study was due to the lower amount of starch per serving (glycaemic load) or due to a change in the intrinsic starch digestibility.

Further studies are needed to better understand how and to what extent sbeII wheat can be used to attenuate the glycaemic potency of different food products. The aim of the present study was to determine the effect of sbeII wheat in bread on postprandial glycaemic response and satiety, compared to WT control bread. Unlike a Glycaemic Index study, this postprandial study was focussed on understanding the effects of sbeII starch in bread on glycaemic response curve size and shape, compared to a conventional white bread, to inform on the potential health effect of replacing conventional white bread with sbeII bread.

The bread rolls were prepared to deliver the same amount of starch per serving (75 g), such that any observed differences in the glycaemic responses to these bread rolls reflect differences in intrinsic starch amylolysis (measured in vitro). A preliminary sensory assessment (palatability) and quality analysis of sbeII bread was also conducted however, due to the small number of participants completing the study, results were not conclusive. The methodology was reported in ESI. $\dagger$

An additional aspect of this study was the measurement of postprandial glucose using a continuous glucose monitoring system (CGM) to assess glycaemic responses, in parallel with fingerprick (FP) sampling for capillary glucose measurements. CGMs were fitted to the upper arm and used enzymaticamperometric technology to measure glucose concentrations in interstitial fluid (IF) over several days. Recent studies have used CGMs to investigate the effect of dietary interventions on glucose responses in healthy individuals. ${ }^{17,18}$ Dye et al. ${ }^{19}$ and Bajka et $a .^{20}$ reported a good correlation between capillary and IF glucose concentrations despite a time lag between measurements, likely due to physiological differences in the regulation of glucose exchange between the blood and the interstitial compartments. In the present study, the relationship between CGM and FP measurements was explored.

\section{Methods}

\section{Bread formulation and process}

A sbeII mutant bread wheat and a WT control bread wheat (Triticum aestivum L. ssp. aestivum) were sown in spring 2018 in a field trial using a randomised block design at the John Innes Centre Church Farm field station (Bawburgh, UK). Wheat was harvested in summer 2018, grains were transported to Campden BRI (Chipping Campden, UK). Following tempering to $16.4 \%$ humidity, grains were debranned and milled on a Bühler mill with feeding rate of $100 \mathrm{~g} \mathrm{~min} \mathrm{~m}^{-1}$, then sieved through $140 \mu \mathrm{m}$ mesh size and passed through a bran finisher fitting a $125 \mu \mathrm{m}$ mesh to produce white flour.

The sbeII and WT control wheat bread rolls were produced using a straight-dough method (AACC Method 10-10.03) at the Quadram Institute (NHS QI Clinical Research Facility, Norwich, UK). The formulation of each bread was adjusted based on the flour's starch content so that both provided the same total starch ( 75 g). Briefly, flour (1217.7 g and $1201.4 \mathrm{~g}$ for sbeII and WT control, respectively), yeast (35.6 $\mathrm{g}$ and $33.9 \mathrm{~g}$ for sbeII and WT control), sugar (44.5 $\mathrm{g}$ and $42.4 \mathrm{~g}$ for sbeII and WT control), salt (22.3 $\mathrm{g}$ and $21.2 \mathrm{~g}$ for sbeII and WT control), shortening (40.7 $\mathrm{g}$ and 38.8 for sbeII and WT control), and water (867.2 $\mathrm{g}$ and $781.6 \mathrm{~g}$ for sbeII and WT control) were mixed at low speed for $3 \mathrm{~min}$ and kneaded for $5 \mathrm{~min}$ at increasing speed, in a heavy-duty planetary mixer with hook attachment (model 5KSM7591XBSM, Kitchen Aid, Antwerp, $\mathrm{BE}$ ). Dough was fermented $2 \mathrm{~h}$ at $21{ }^{\circ} \mathrm{C}$ then portioned into rolls and proofed for $15 \mathrm{~min}$ at $38{ }^{\circ} \mathrm{C}$ with $100 \%$ relative humidity, then baked using a combination oven (Rational Self Cooking Centre, Luton, UK) for $15 \mathrm{~min}$ at $185{ }^{\circ} \mathrm{C}$ (40\% humidity for $10 \mathrm{~min}$ and $10 \%$ humidity for the last $5 \mathrm{~min})$. Baked rolls reached $\sim 95{ }^{\circ} \mathrm{C}$ core temperature. After $2 \mathrm{~h}$ cooling at $21{ }^{\circ} \mathrm{C}$, breads were packed in resealable, opaque polyethylene bags ( $3 \mathrm{~mm}$ thickness) and stored at $-20^{\circ} \mathrm{C}$. Breads were produced in four batches of 12 rolls (48 rolls per type of flour), under identical conditions and paired by their baking position in the oven. Rolls were thawed for $16 \mathrm{~h}$ (overnight) and either served to human study participants as part of their breakfast meal or used for in vitro analyses. Breads were consumed within three months of manufacture.

\section{Bread characteristics and in vitro amylolysis}

Nutrient composition and microbiological safety. Two pairs of bread rolls (sbeII and WT control) were randomly selected for proximate nutritional analysis and microbiological safety testing before the start of the study. These were performed by UKAS accredited testing at ALS Laboratories Ltd, Chatteris, Cambridgeshire, UK, as described by Bajka et al. (2021). ${ }^{20}$ Bread rolls were deemed safe for human consumption; details can be found in ESI Table 1. $\dagger$

Starch characteristics. The proportion of digestible and resistant starch in flour were determined using a 'Total starch kit' (KTSTA-100A KOH format, AOAC 996.11, Megazyme International, Wicklow, Ireland), $n=8$. Starch isolation and apparent amylose determination $(n=3)$ were carried out as described previously, Corrado et al. 2020. ${ }^{14}$

Five pairs of each bread type were randomly selected for in vitro analysis of starch characteristics. From each roll, a minimum of three technical replicates were sampled for analysis, as specified below. Rolls were left to thaw in their packaging at room temperature $\left(\sim 21{ }^{\circ} \mathrm{C}\right)$ for $16 \mathrm{~h}$, then were blended in a food processor (Kenwood $\mathrm{CH} 180$ Mini chopper) for 50 seconds and sieved to obtain a $1 \mathrm{~mm}$ fraction, used for moisture determination and in vitro amylolysis assay, and a $500 \mu \mathrm{m}$ fraction, used to measure digestible and resistant starch. These fractions were weighed into dry-tared aluminium pans 
in triplicates (technical replicates) for moisture determination, into $2 \mathrm{~mL}$ safe lock tubes (STARLAB (UK), Ltd) in quadruplicates (technical replicates) for starch measurements, and into $15 \mathrm{~mL}$ Corning centrifuge tubes (Merck KGaA, Darmstadt, DE) in triplicates (technical replicates) for amylolysis analysis. Digestible and resistant proportions of starch were measured using the reagents from 'Total starch kit' and 'Resistant starch kit' K-RSTAR (AOAC 2002.02, Megazyme International, Wicklow, Ireland), using a small-scale version of the method, as described by Edwards et al., 2015. ${ }^{21}$ Moisture was determined using the AACC (44-15A) air oven method, one stage procedure.

In vitro starch susceptibility to amylase. Starch susceptibility to amylase digestion (amylolysis) was determined after five months of storage using an established amylolysis assay method based on enzyme-kinetic principles, on five bread rolls per type, three technical replicates. Full details of this method, which involves incubation of starch-rich food material with amylase, quantification of amylolysis products by PAHBAH, and calculation of starch digestibility indices, were recently published. ${ }^{22}$ Briefly, products of amylolysis were collected after $0,3,6,9,12,15,18,21,25,30,45,60,75,90 \mathrm{~min}$ of incubation with porcine pancreatic $\alpha$-amylase, as in a previous study on sbeII semolina pudding, ${ }^{14}$ and reducing sugars (maltose) were then quantified by the 'PAHBAH' ( $p$-hydroxybenzoic acid hydrazide) assay. The resulting amylolysis curves were then baseline corrected by subtracting the value at $t=$ $0 \min \left(Y_{0}\right)$ from each replicate. Starch digestibility was expressed as percentage of starch digested $C_{t}$, and $C_{90}$ representing extent of digestion after 90 min of incubation with $\alpha$-amylase. Values for the first-order rate constant, $k$ and endpoint, $C_{\infty}{ }^{23}$ were obtained using a non-linear regression model to fit a first order equation $C_{t}=C_{\infty}\left(1-e^{-k t}\right)$ to the experimental data, using the stats package in Rstudio. ${ }^{24,25}$ Parameters were compared using independent groups $t$-tests, values reported throughout are means $\pm \operatorname{SEMs}(n=5)$ unless otherwise specified.

Bread roll quality assessment. Four pairs of bread rolls (sbeII and WT control) were randomly selected for quality assessment which included specific volume, crumb and crust colour, and texture. The rolls were left to thaw in their packaging at room temperature $\left(\sim 21^{\circ} \mathrm{C}\right)$ for $16 \mathrm{~h}$. Bulk density $\left(\mathrm{g} \mathrm{cm}^{-3}\right)$ was determined using the rapeseed displacement method (AACC method 10-05.01); a metal block $(14 \times 6.3 \times 5 \mathrm{~cm})$ was used as the standard. Mass was determined immediately prior to rapeseed displacement measures on an OHaus (Model Explorer, NJ, USA) scale to $0.05 \mathrm{~g}$ precision. The specific bread volume $\left(\mathrm{cm}^{3} \mathrm{~g}^{-1}\right)$ was determined as the volume/weight ratio of baked breads.

Crumb texture and bread colour were measured on crust and crumb. Texture was measured instrumentally using a 'twobite test' $(n=4)$ on a TA-XT2 Texture Analyser (Stable Micro Systems, Godalming, UK), ${ }^{20}$ equipped with a five $\mathrm{kg}$ load cell using a modified AACC method 74-09. The Texture Analyser was equipped with a $50 \mathrm{~mm}$ diameter compression plate (P50); a uniaxial compression with crosshead speed of $100 \mathrm{~mm}$ $\min ^{-1}$ was applied to $25 \times 25 \times 25 \mathrm{~mm}$ samples to mimic mastication, with crumb hardness corresponding to the force $(N)$ required for $40 \%$ compression. Exponent (version 6.0, Stable Micro Systems, Godalming, UK) software for texture profile analysis was used to assess the following texture parameters: hardness, springiness, cohesiveness, gumminess, chewiness and resilience. Four independent measurements of colour were taken for each bread roll type, using a 'Gretag Macbeth' twenty-four patch Colour Checker as reference. ${ }^{26} \mathrm{Hue}$ angle and Chroma were calculated using the $L^{*}, a^{*}, b^{*}$ parameters. ${ }^{27}$ Parameters were compared using independent groups $t$-tests, values reported throughout are means $\pm \operatorname{SEMs}(n=4)$ unless otherwise specified.

\section{Acute postprandial intervention study}

Ethical review. The REST study protocol procedures received favourable ethical opinion by the Health Research Authority England (South Cambridge Ethics Committee, REC reference 19/EE/0260, IRAS 262271) and was registered with ClinicalTrials.gov (NCT04197726). The study was also approved by the Human Research Governance Committee of the Quadram Institute and by the Department of Research and Development (R\&D) of the Norfolk and Norwich University Hospitals NHS Foundation Trust (NNUH, reference 125-07-19). All participants provided written informed consent to take part in this study. All participants' data were stored in accordance with the General Data Protection Regulation 2018 and biological samples were handled, stored, transported, and disposed of in accordance with the Human Tissue Act (2004).

Study design. We conducted a two arm two period doubleblind randomised cross-over study comparing the effect of sbeII bread and WT control bread on glycaemic response, measured in capillary blood by FP test and in IF by CGM.

Enrolled participants were randomised in blocks of four stratified by sex to complete 3 visits: Visit 1, to apply the CGM sensors, followed by Visit 2 and Visit 3, where participants consumed either sbeII or WT control bread on consecutive occasions, in randomised order, with a minimum of four days washout period between visits with no dietary restriction. During intervention visits, capillary blood was obtained by fingerprick test to determine glycaemic response and in parallel, by CGM to measure glycaemic response in IF. The effect of sbeII bread consumption on satiety was determined using a visual analogue scale questionnaire $(\mathrm{VAS})^{28}$ before and after bread consumption as well as changes in energy intake during the subsequent meal (satiety challenge).

Between interventions, participants completed a non-consecutive three-day weighted food diary, on two weekdays and one weekend day, to capture habitual diet. The study lasted approximately one month from screening to follow up.

A priori power calculation. The power calculation was carried out using an $\mathrm{R}$ core function, power.t.test. ${ }^{24}$ It was hypothesised that sbeII bread, with lower susceptibility to hydrolysis in vitro than the WT control, would induce a lower glucose response compared to the WT control in vivo (iAUC 0120). A sample size of 19 was required to detect a $20 \%$ relative 
difference in iAUC difference with $80 \%$ power, assuming a $28.7 \mathrm{mmoL} \mathrm{L}{ }^{-1} \mathrm{~min}^{-1} \mathrm{SD}$ between pairs as observed in a previous study by Rosén et al. 2009. ${ }^{29}$ Planned sample size was increased to 25 to account for potential dropouts. Ultimately, owing to COVID restrictions, only eight participants could complete the study.

Study participants. Participants were recruited within 40 miles of the Norwich Research Park, Norfolk, UK. Participants meeting the following criteria were deemed eligible to take part: age 18-65 years, BMI $18-25 \mathrm{~kg} \mathrm{~m}^{-2}$, fasting glucose $<6.1 \mathrm{mmoL} \mathrm{L}{ }^{-1}, \mathrm{HbA1}_{\mathrm{c}}<42 \mathrm{mmoL} \mathrm{L}^{-1}$, blood pressure $<160 / 100 \mathrm{mmHg}$. Participants were excluded if they were smokers, allergic or intolerant to the study foods or to adhesives, were alcohol or substance abusers or had insulindependent or non-insulin dependent diabetes, gastrointestinal disorder, anaemia, cardiovascular disease, certain cancers, or unstable body weight during the past 3 months. Women who were pregnant, lactating, or had given birth during the last 12 months were also excluded.

Study visits. The study consisted of three visits.

During Visit 1, participants applied two CGM Freestyle Libre sensors (Abbott Diabetes Care, Alameda, CA) to record IF glucose continuously for 14 days. They then observed a twoday CGM calibration period during which no interventions or restrictions were applied. Then, they completed Visit 2 and Visit 3 as follows: after a standardised evening meal and $\sim 12 \mathrm{~h}$ fast, baseline measurements of capillary blood glucose and satiety were taken while CGM sensors recorded IF glucose concentrations. They were then given the intervention meal as their first meal of the day (breakfast), either sbeII or WT control bread roll, after which glycaemic, sensory (palatability) and satiety responses (VAS) were measured over $3.5 \mathrm{~h}$. Four hours after breakfast, a lunch meal was provided ad libitum as satiety challenge. At the end of the satiety challenge, the visit was considered completed and participants left the Clinical Research Facility of the Quadram Institute. A follow up questionnaire was completed the day after Visit 3 to capture participants' experience wearing and using CGM devices.

Study meals. The standard evening meal consumed the night before Visit 2 and 3 consisted of a ready meal chosen from a pre-selected list of ready meals with similar nutrient composition. The ready meals contained $\sim 365 \mathrm{kcal}, \sim 39 \mathrm{~g}$ available carbohydrate, $\sim 8 \mathrm{~g}$ sugars, $\sim 8 \mathrm{~g}$ dietary fibre, $\sim 17 \mathrm{~g}$ protein, based on the nutrient declaration on food packaging. Only water was allowed between the evening meal and the intervention breakfast.

The intervention breakfast consisted of one bread roll providing $\sim 75 \mathrm{~g}$ of total starch, either sbeII or WT control, $10 \mathrm{~g}$ of low-fat spread (Flora Dairy Free) and approximately $250 \mathrm{~mL}$ of water to drink. The liquid component of the breakfast meal was standardised by adjusting the water drink to the water content of each bread. The total water content each meal was approximately $317 \mathrm{~g}$, the average serving size of sbeII breads was $153.19 \mathrm{~g} \pm 0.49 \mathrm{~g}$ (fresh weight) and the average size of WT control breads was $147.28 \mathrm{~g} \pm 0.73 \mathrm{~g}$ (fresh weight), (mean \pm SEMs, $n=8)$.
The lunch meal (satiety challenge) served ad libitum was made of portions of $80 \mathrm{~g}$ white rice, served with either 'Option A' $85 \mathrm{~g}$ of tomato and basil pasta sauce and $7 \mathrm{~g}$ of parmesan cheese or 'Option B' $90 \mathrm{~g}$ of beef Bolognese pasta sauce, and was served with $250 \mathrm{~mL}$ of water. Both sauce options had a similar nutrient composition and provided approximately $177.2 \mathrm{kcal} \pm 0.7 \mathrm{kcal}, 27.3 \mathrm{~g} \pm 0.4 \mathrm{~g}$ carbohydrate of which, $4.6 \mathrm{~g} \pm 0.9 \mathrm{~g}$ sugars, $1.2 \mathrm{~g} \pm 0.2 \mathrm{~g}$ fibre, $6.9 \mathrm{~g} \pm 0.8 \mathrm{~g}$ protein per serving (average of two options $\pm \mathrm{SD}$, calculated from the nutrient declaration on food packaging). Participants were asked in advance to choose from sauce option A and B and consumed the same lunch meal during both visits.

Satiety challenge: ad libitum lunch. Four hours after consuming the intervention meal, participants consumed an ad libitum lunch to determine the effect of the intervention on subsequent energy intake (kcal). New bowls of rice with the sauce of choice (one portion) were provided at specific times depending on the speed of consumption of each participant (approximately every 3-10 $\mathrm{min}$ ), to ensure that warm food was served and that finishing a bowl did not act as a cue to stop eating. Participants were instructed to eat until they were "comfortably satisfied". Satiety changes were estimated based on VAS responses following bread consumption and the total energy intake, measured as the 'kcal', during the subsequent lunch ad libitum.

\section{Data and statistical analysis}

Analysis of glycaemic response measures. Glucose concentrations measured in capillary blood by FP and in IF by CGM at pre-selected time points were used to compare the postprandial glycaemic responses to sbeII and WT wheat bread rolls.

Baseline fasting glucose was obtained 15, 10 and $5 \mathrm{~min}$ before the breakfast intervention meal by FP and at 30, 15 and 0 min before the breakfast intervention meal by CGM. The average of these three measurements was used as T0.

Intervention meals were consumed within $20 \mathrm{~min}$, followed by ten pricks to measure capillary blood glucose concentrations, every $15 \mathrm{~min}$ for $3.5 \mathrm{~h}$ while glucose concentration in IF was recorded continuously every $15 \mathrm{~min}$ by the two sensors worn by each participant. Data up to $3.5 \mathrm{~h}$ was collected in order to capture the end of the postprandial response (return to baseline) to these study foods.

The glycaemic response to breads (iAUC) was calculated geometrically between 0 and $210 \mathrm{~min}$ (primary outcome), by applying the trapezoid rule to the area over the fasting glucose baseline and under the curve, as recommended by Brouns et $a l .{ }^{30}$ Average glucose peak and glucose dip were calculated as the maximum (peak) and minimum (dip) glucose concentrations achieved after each meal; for capillary glucose this was calculated between 15 and $210 \mathrm{~min}$, for IF glucose, between 15 and $240 \mathrm{~min}$. Time to peak was calculated as the time to reach the maximum glucose concentration following the meal.

Statistical models. Capillary and IF glucose iAUCs were compared across groups using a mixed-effects model using the lmer package v. 3.1.2 $2^{31}$ in Rstudio, with bread type as fixed effect and a random intercept per participant. The model used 
to obtain the IF effect estimates also included the visit nested within participant, as an additional random intercept to account for having data from two CGM sensors. Estimates of effect were obtained with Satterthwaite approximations for degrees of freedom, using the emmeans package v. 1.4..$^{32}$ in Rstudio and Pearson pairwise correlation (within participant, between sensors) was determined using the stats package previously mentioned.

Datasets were curated using package reshape v. 1.4.3, graphs and plots were made using ggplot2 v. 3.3.1 and ggpmisc v. 0.3.5 $5^{33-35}$ in Rstudio.

Analysis of satiety and sensory measures. VAS questionnaires were completed before the meal (T0), and at 30, 90, 180 and 270 min post-prandially to determine changes in hunger, fullness, desire to eat, and prospective consumption. The scales consisted of horizontal lines $100 \mathrm{~mm}$ long, anchored by "not at all" and "extremely" at opposite ends. Satiety VAS questionnaires were scanned using ImageJ (NIH, USA), ${ }^{36}$ and an appetite score was calculated as follows: appetite score $=[$ desire to eat + hunger $+(100-$ fullness $)+$ prospective consumption $] / 4$ as described by Anderson et al. (2010). ${ }^{37}$

\section{Results}

\section{Flour characteristics}

The sbell and WT control flours had similar total starch (73.9 \pm $1.2 \mathrm{~g} v s .74 .94 \pm 0.8 \mathrm{~g}$ per $100 \mathrm{~g}$ of flour, means \pm SEMs, $n=8$ ). Apparent amylose (means \pm SEMs, $n=3$ ) was higher in sbell starch $(39 \pm 1.1 \%$ of total starch) than in WT control starch $(25.7 \pm 0.7 \%$ of total starch), hence the proportion of resistant starch (means \pm SEMs, $n=8$ ) was much higher in sbell flour $(6.4 \pm 0.5 \%$ of total starch) compared to WT control (0.6 \pm $0.1 \%$ of total starch). Indicators of performance in the field and extraction rate can be found in ESI Table $2 . \dagger$

\section{Bread characteristics}

Breads were formulated to deliver approximately $75 \mathrm{~g}$ of total starch per serving. Nutrient composition, indicators of in vitro amylolysis and starch characteristics of bread rolls are reported in Table 1. Starch in sbeII bread was less susceptible to amylolysis compared to the WT control $\left(C_{90}\right.$ mean difference $=$ $13.03 \%$ of starch digested, 95\%CI [1.91, 24.15], independent groups $t$-test, $p$-value $=0.02)$. First order equations fit starch digestion curves well, Table 1 . The sbeII bread was characterized by a lower starch breakdown after $90 \mathrm{~min}$ of hydrolysis and a lower digestion rate $(k)$, Fig. $1 \mathrm{~A}$.

Hardness, gumminess, chewiness and cohesiveness were significantly higher in sbeII bread rolls compared to WT control breads while there were no differences in springiness and in resilience between bread types (Fig. 1B.1 and B.2). The bulk density of sbeII bread was greater than the WT control but no differences in volume were detected (Fig. 1B.3 and B.4). Crust colour varied between sbeII and WT breads with a darker colour and a matte finish for the sbeII bread crust, compared to the WT control (Fig. 1C), ESI Table $3 . \dagger$
Table 1 Compositions of the morning intervention meal with either sbell or WT control bread. Bread and starch characteristics measured at the end of recruitment $(5$ months after production and storage at $\left.-20^{\circ} \mathrm{C}\right)$

\begin{tabular}{|c|c|c|}
\hline Nutrients per serving & $\begin{array}{l}\text { WT control bread } \\
\text { meal }\end{array}$ & $\begin{array}{l}\text { sbeII bread } \\
\text { meal }\end{array}$ \\
\hline Energy $^{a}(\mathrm{kcal})$ & 438.7 & 430.8 \\
\hline Energy (kJ) & 1854.9 & 1815.7 \\
\hline $\operatorname{Protein}^{b}(\mathrm{~g})$ & 12.15 & 12.96 \\
\hline Total fat ${ }^{c}(\mathrm{~g})$ & 11 & 11.54 \\
\hline Saturated fat $(\mathrm{g})$ & 2.92 & 3.07 \\
\hline Poly-unsaturated fat (g) & 3.54 & 3.77 \\
\hline Ash & 2.19 & 2.29 \\
\hline $\begin{array}{l}\text { Available carbohydrates }{ }^{d} \\
\text { (g) }\end{array}$ & 70.9 & 65.1 \\
\hline Total sugars $(\mathrm{g})$ & 2.92 & 3.06 \\
\hline Fibre $\mathrm{AOAC}^{e}(\mathrm{~g})$ & 4.53 & 7.5 \\
\hline Bread characteristics & WT bread & sbeII bread \\
\hline Bread roll (g) & $146.24 \pm 2.55$ & $153.24 \pm 0.38^{* * *}$ \\
\hline Moisture (\%) & $38.59 \pm 0.17$ & $42.13 \pm 1.02^{* * *}$ \\
\hline Total starch $(\mathrm{g})$ & 74.9 & 74.9 \\
\hline Digestible starch (\%) & $96.5 \pm 0.2$ & $91.8 \pm 0.8^{* * *}$ \\
\hline Resistant starch (\%) & $3.5 \pm 0.1$ & $8.2 \pm 0.2^{* * *}$ \\
\hline In vitro amylolysis & WT bread & sbeII bread \\
\hline$C_{90}(\%)$ & $91.3 \pm 2.9$ & $78.3 \pm 3.7^{*}$ \\
\hline$C_{\infty}(\%)$ & 91.6 & 85.4 \\
\hline$k\left(\min ^{-1}\right)$ & 0.048 & 0.030 \\
\hline$r^{2}$ & 0.99 & 0.99 \\
\hline Sum of squares & 1706 & 767 \\
\hline
\end{tabular}

Bread roll weight is reported as the mean \pm SEMs of 8 rolls measured after $16 \mathrm{~h}$ thaw. All other bread characteristics were measured on the same bread rolls, after $16 \mathrm{~h}$ thaw. Moisture and in vitro amylolysis experimental values are reported as the mean \pm SEMs of 5 bread rolls; digestible and resistant proportion of starch are reported as mean \pm SEMs, 4 bread rolls. Total starch content of breads is based on the flour starch content. The bread formulation was adjusted to match total starch content of sbeII and WT control breads. Parameter values that are significantly different in the sbeII bread compared to the WT control bread are indicated with an asterisk; ${ }^{*} P<0.05,{ }^{* *} P<0.01$ and ${ }^{* *} P<0.001$ (independent groups $t$-test). ${ }^{a}$ Calculated using standard energy conversion factors (EC 2008/100 and 90/496). ${ }^{b}$ Nitrogen-toprotein conversion factor $=6.25 .{ }^{c}$ Determined by NMR. ${ }^{d}$ Determined by ion exchange chromatography, calculated by difference. ${ }^{e} \mathrm{AOAC}$ method 985.29 ('Prosky method'), which includes RS type 3 (retrograded starch).

\section{Acute postprandial intervention study}

Only 8 out of 19 enrolled participants completed the study due to the COVID-19 outbreak and consequent lockdown (Fig. 2). Their characteristics are reported in Table 2. All other baseline measurements are reported in ESI Table $4 . \dagger$ The average energy intake recorded by participants during the three-day food diary was within the UK dietary recommendations ${ }^{38}$ and their carbohydrate intake was approximately $41 \%$ of their total kcal intake (men and women), predominantly derived from starch Table 2. More details about the average dietary intake of study participants can be found in ESI Table $5 . \dagger$

Glycaemic response. Participants required approximately 8-10 min to consume the intervention meals. The estimates of 

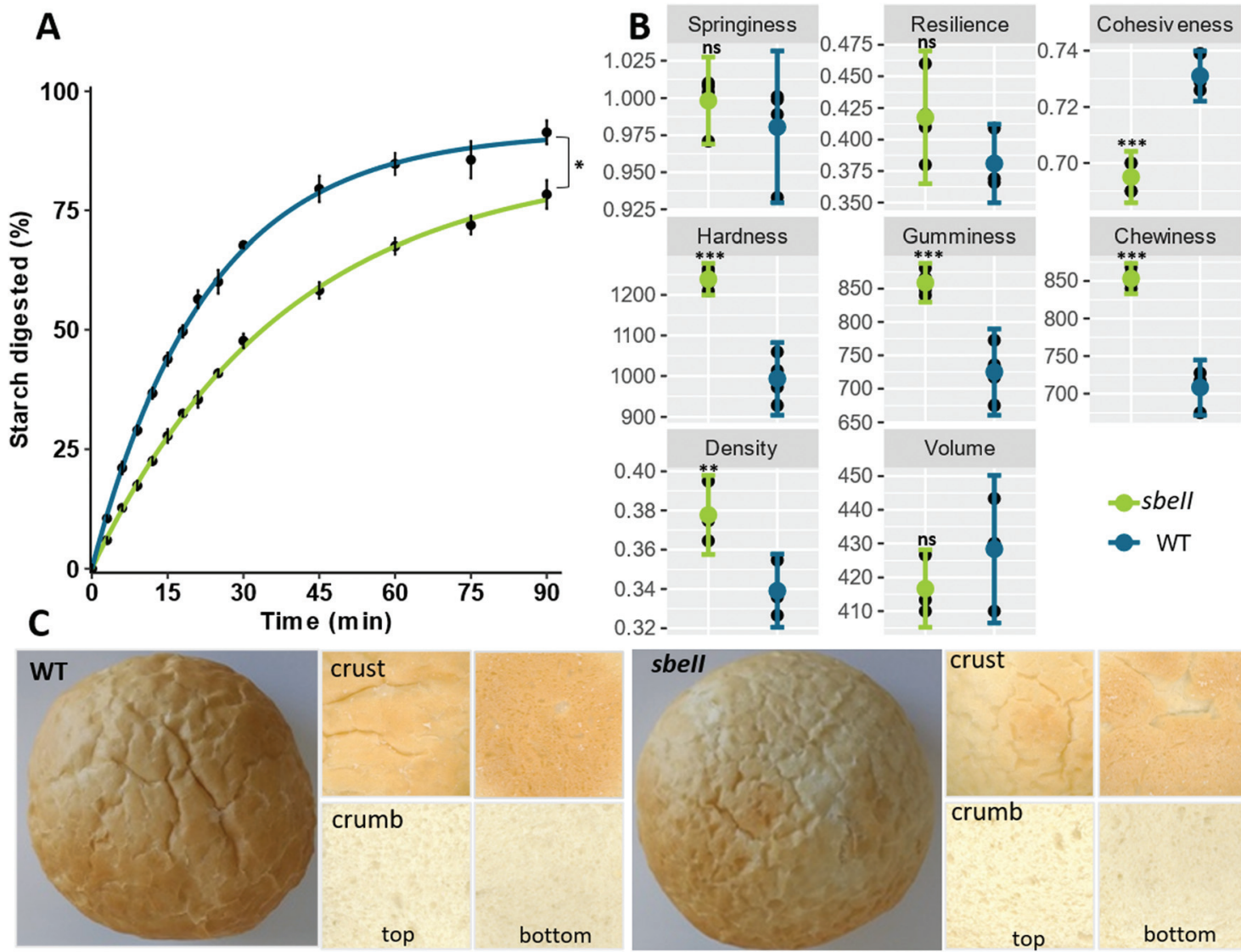

Fig. 1 In vitro characteristics of sbell (green) and WT (blue) bread rolls after 5 months of storage at $-20^{\circ} \mathrm{C}$. (A) Starch amylolysis; each experimental data point represents the mean value from the analysis of five independent samples with error bars $= \pm \mathrm{SEMs}, n=5$. Experimental data are shown by fitting a first-order equation based on the $k$ and $C_{\infty}$ values obtained from a non-linear regression model. (B) Bread quality parameters, mean \pm SEMs, $n=4$; bread crumb texture analysis on Texture Analyser showing springiness, cohesiveness, resilience (\%), and hardness (Newtons), gumminess (AU) chewiness (Newtons per s); bread volume and density (bulk density). (C) Images of bread rolls as whole, crust and crumb.

effects of sbeII and WT control breads on postprandial glucose responses are reported in Table 3.

Glycaemic response (iAUC) to sbell bread was $22.9 \mathrm{mmoL}$ $\mathrm{L}^{-1} \mathrm{~min}^{-1}, 95 \%$ CI $[-13.6,59.5]$, lower than control bread (corresponding to an average $15.7 \%$ reduction following sbeII bread compared to WT control bread). Similarly, the effect size measured in IF by CGM was $19.2 \mathrm{mmoL} \mathrm{L}^{-1}$ $\min ^{-1}(14.8 \%$ difference between sbeII and WT control breads). While these estimates from only 8 participants are not statistically significant, they are consistent with the in vitro findings and our anticipated effect size of $20 \%$ difference between groups. Participants' fasting glucose concentrations measured in capillary blood and IF were within normal range and their fasting glucose values did not vary significantly between the two visits (FP fasting glucose $p$-value $=0.58$, IF fasting glucose $p$-value $=0.62$, paired $t$-test). The overall pattern of postprandial capillary blood glucose was similar after the consumption of sbeII bread and WT control (Fig. 3A and B). Fig. 3C shows the incremental glucose peak (iCmax) and Fig. 3D shows the average capillary glucose responses $\left(\mathrm{iAUC}_{0-120}\right)$. Other postprandial capillary glucose indicators are reported in Table 3. Time to peak calculation from the start of the test showed that the IF measurements lagged $11 \mathrm{~min}$ compared to capillary blood glucose.

Satiety responses. Participants indicated that their 'desire to eat' and 'hunger' were similar after consuming sbeII and WT control bread, as measured by the satiety VAS questionnaires, Fig. 3E. There is some evidence of increased 'fullness' up to 30 min after consuming sbeII bread (paired $t$-test, mean difference $=2.16,95 \% \mathrm{CI}[0.65,3.6], p$-value $=0.01)$. Plots showing individual satiety responses can be found in ESI Fig. $1 . \dagger$ The increased 'fullness' appeared to be temporary and did not seem to affect the following meal (lunch), as subsequent energy and water intake ad libitum (lunch satiety challenge) did not differ following the two intervention meals (energy intake $=1131.04 \pm 154 \mathrm{kcal}$ and $1076.72 \pm 130$ after consuming sbeII and WT control breads respectively, means \pm SEM, $n=8$, and water intake $=402 \mathrm{~mL} \pm 90 \mathrm{~mL}$ and $400 \mathrm{~mL} \pm 56 \mathrm{~mL}$ after sbeII and WT control breads respectively, means \pm SEM, $n=8$ ), Fig. 3F.

Comparing FP and CGM measures of postprandial glycaemia. Each participant wore two identical CGM sensors for all intervention visits on the same arm. Two participants lost one 


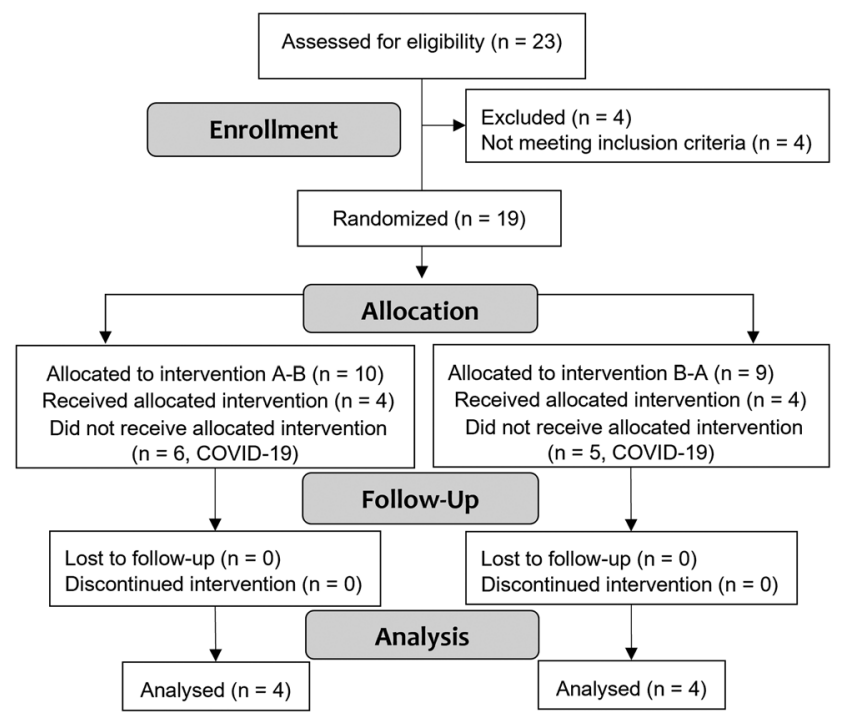

Fig. 2 CONSORT diagram of the REST study. Interventions were blinded to the participants and the study team using an allocation concealment. At the end of recruitment, breads were grouped as ' $A$ ' or ' $B$ ' to allow the study team to complete the data analysis. Researchers were unblinded once the data analysis was completed. Two participants lost one sensor after intervention 1 and completed intervention 2 with one sensor only. Two participants lost one sensor before intervention 1 and had it replaced.

Table 2 Screening characteristics and habitual dietary intake of study cohort (inclusion/exclusion criteria)

\begin{tabular}{|c|c|c|c|c|}
\hline Characteristics & Mean & SD & Min & Max \\
\hline Age (years) & 33 & 13.6 & 23 & 58 \\
\hline Height (m) & 1.73 & 8.6 & 1.60 & 1.90 \\
\hline Weight (kg) & 69.8 & 10.4 & 58.1 & 91.9 \\
\hline $\operatorname{BMI}\left(\mathrm{kg} \mathrm{m}^{-2}\right)$ & 23.2 & 1.7 & 20.7 & 25.4 \\
\hline Venous fasting glucose $\left(\mathrm{mmoL} \mathrm{L}^{-1}\right)$ & 4.4 & 0.2 & 4.1 & 4.7 \\
\hline $\mathrm{HbA1c}\left(\mathrm{mmol} \mathrm{mol}^{-1}\right)$ & 34.4 & 2.0 & 31.0 & 36.0 \\
\hline Three-days average intake & Men & & \multicolumn{2}{|c|}{ Women } \\
\hline Energy (kcal per day) & \multirow{2}{*}{\multicolumn{2}{|c|}{$2780.5 \pm 735.9$}} & \multicolumn{2}{|c|}{$1989 \pm 430.9$} \\
\hline Starch (g per day) & & & \multirow{2}{*}{\multicolumn{2}{|c|}{$\begin{array}{l}136.8 \mathrm{~g} \pm 39.8 \\
18.7 \mathrm{~g}+3.1\end{array}$}} \\
\hline Non-starch polysaccharide ( $\mathrm{g}$ per day) & \multicolumn{2}{|c|}{$24.90+3.3$} & & \\
\hline
\end{tabular}

$N=8$ of which 4 males and 4 females. Diet intake is reported as mean \pm SDs, $n=4$ men and $n=4$ women.

sensor after intervention 1 and completed intervention 2 with one sensor only. Two other participants lost one sensor before intervention 1 and had it replaced. Based on the follow up questionnaire completed by the study participants, the experience of wearing two CGM sensors was described as "quick" and "easy", some participants found the application slightly painful "like a jab" however, most described them "comfortable" to wear and "interesting".

Intra-individual variation (CV\%) between the two sensors used in this study by each participant ranged $0-17 \%$ during postprandial breakfast phase and $0-22.7 \%$ during the postprandial lunch phase. There was a strong correlation between the two

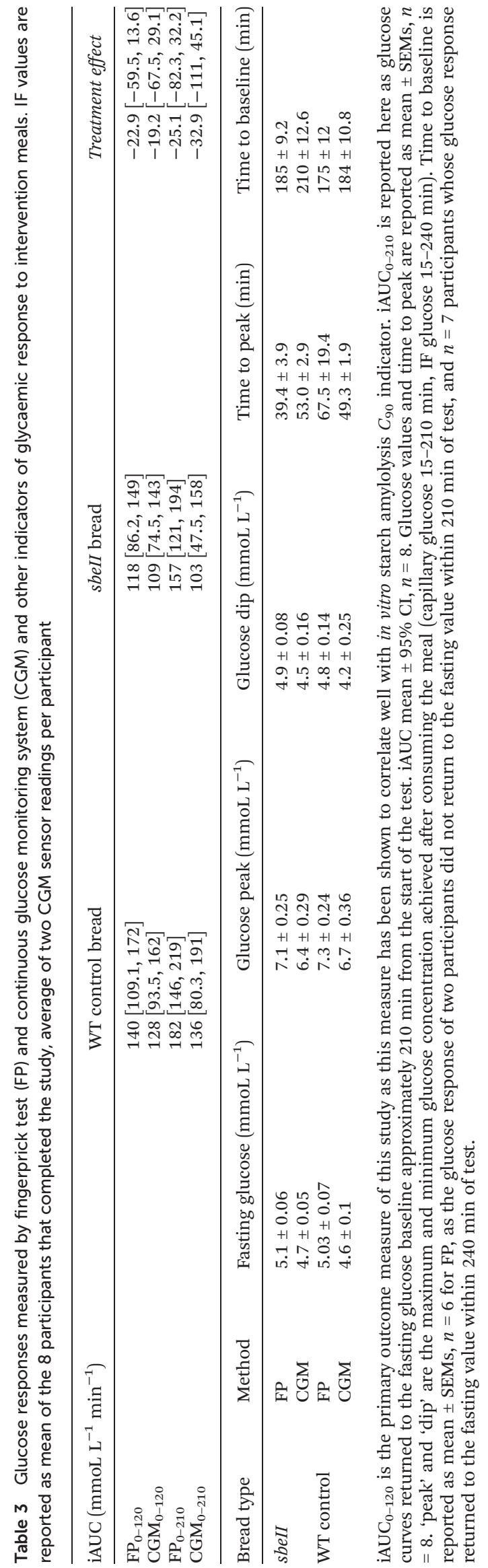


A
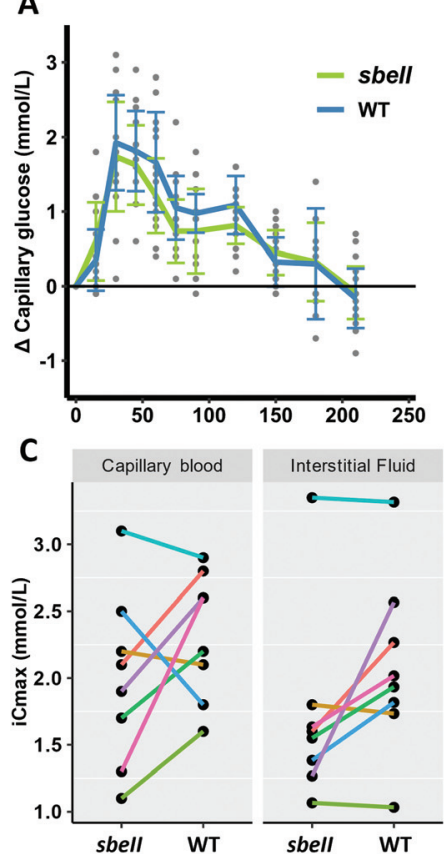

B

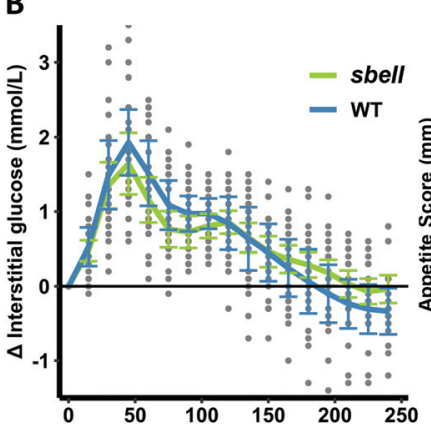

D

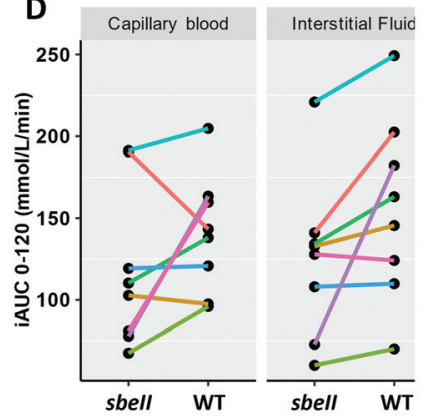

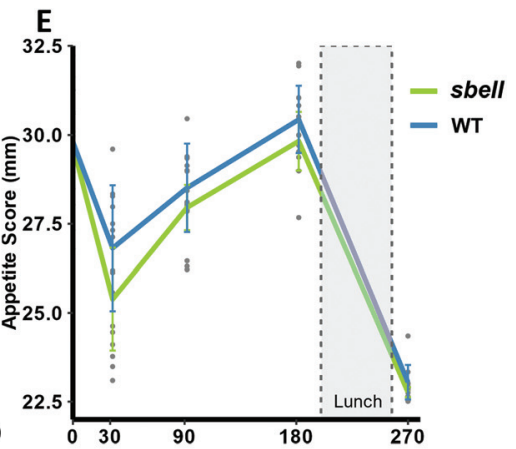

F Energy (kcal) Water $(\mathrm{mL})$

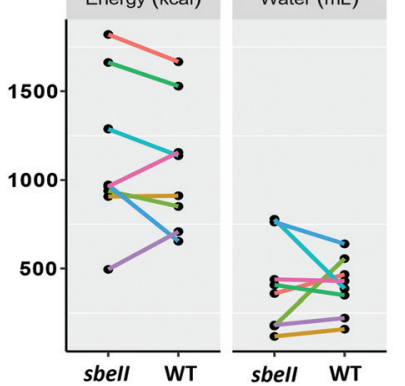

Fig. 3 Post-prandial glucose concentrations. Incremental glucose concentrations measured in capillary blood (A) and IF (B). Datapoints represent individual glucose values, curves are mean glucose profile $(n=8)$ and error bars $= \pm$ SEMs. C. Incremental maximum concentration of glucose (iCmax) in capillary and IF. (D) Individual incremental glucose over time detected in capillary blood and IF (iAUC $\mathrm{C}_{0-120}$ ). (E) Change in appetite score from fasting until after the ad libitum lunch (satiety challenge), score calculated as [desire to eat + hunger $+(100-$ fullness $)+$ prospective consumption] $/ 4, n=8$ and error bars $= \pm$ SEMs. (F) Energy and water intake during satiety challenge following the intervention meals $(n=8)$.
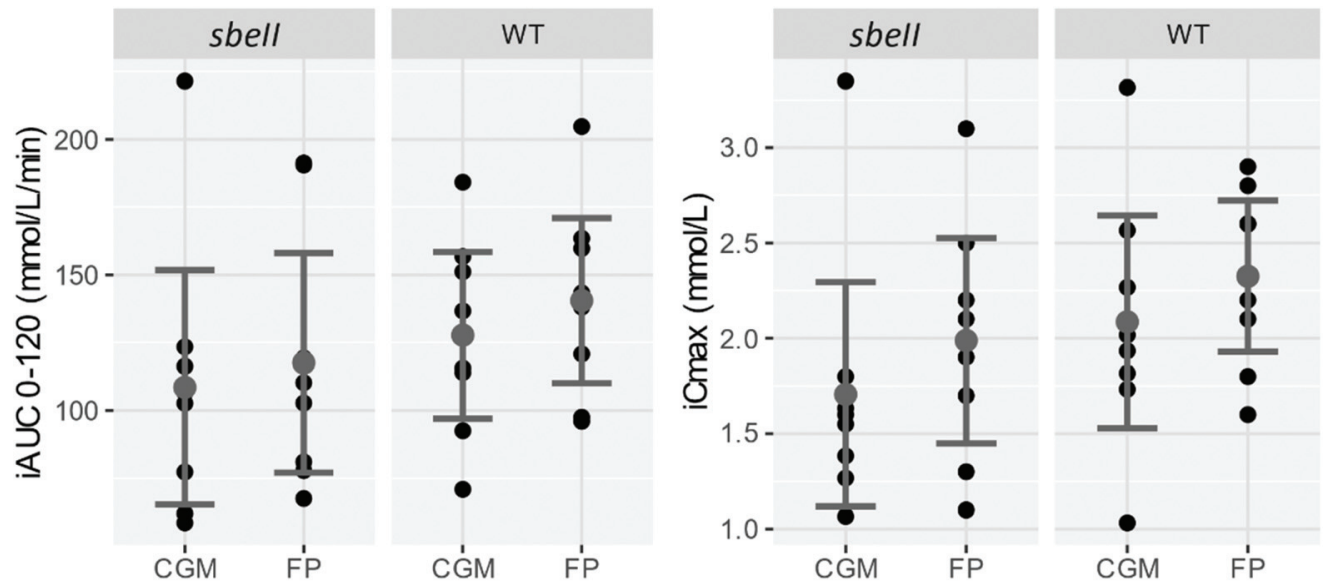

Fig. 4 iAUC and iCmax measured by CGM in IF and in capillary blood by FP after consuming sbell and WT control bread, respectively. Data points represent individual responses (black), the mean of eight subjects in grey with error bars $= \pm$ SEMs.

CGM sensors worn by each participant $(r=0.91)$; on average, there was a $10.5 \mathrm{mmol} \mathrm{L}{ }^{-1} \mathrm{~min}^{-1}$ difference between sensors' measurements of glycaemic response (iAUC 0-120) within participant. We observed a $5.25 \mathrm{mmol} \mathrm{L}^{-1} \mathrm{~min}^{-1}, 95 \%$ CI $[-11.3,21.8]$ difference between sensors after consuming sbeII bread $(n=8)$ and $17.6 \mathrm{mmol} \mathrm{L}{ }^{-1} \mathrm{~min}^{-1}, 95 \%$ CI $[-5.73,40.9]$ after WT bread $(n=6)$. Glucose response curves are shown in ESI Fig. $3 . \dagger$

Overall, the glucose response measured by FP and by CGM was similar (iAUC and iCmax), Fig. 4.

\section{Discussion}

This study investigated the use of a sbeII mutant wheat with a higher amylose proportion for production of slowly digested and/or low glycaemic bread products, compared to conventional wheat starch. Bread rolls made from sbeII mutant wheat contained more RS and showed lower susceptibility to amylase digestion that the WT control, which is consistent with the hypothesis that the presence of mutations in 
sbeII genes lead to wheat foods with increased starch resistance to digestion. ${ }^{14}$

Previous studies measuring glycaemic potency of highamylose food products reported conflicting results. Often, this is derived from inconsistent carbohydrate content across test meals; Behall et al. in $2002^{39}$ presented a very good overview of the different studies that compared glycaemic responses to high and low amylose food products and showed that different methods used to determine the RS and dietary fibre can contribute to the variability in glycaemic measurements. In this study, we report amylose and RS portion of starch in bread rolls, as well as starch resistance to digestion measured using a single-enzyme system that allowed us to determine the rate and extent of starch digestion, previously shown to correlate with glycaemic index measured in vivo. ${ }^{22}$

Here, we showed that the predicted difference in glycaemic response between sbeII and WT control breads from in vitro amylolysis experiments is consistent with the observed difference in glucose concentrations after consumption of sbeII and WT control breads, although our estimate of the effect in vivo is imprecise because only around one third of the planned sample could be completed.

Studies using novel wheat genotypes characterized by a high amylose content have consistently reported a lowering effect on postprandial glucose response. Previously, Belobrajdic et al. ${ }^{16}$ reported a lower glycaemic response to high amylose wheat bread measured in plasma glucose. The low glycaemic effect was likely due to the reduced amount of carbohydrate provided in the high-amylose bread compared to the control bread. In this study, we showed that sbeII bread has potential to elicit lower glycaemic response compared to a conventional white bread without changing the amount of starch provided.

Hallström et al. $(2011)^{43}$ showed that bread made from a high-amylose wheat (38\% of starch) induced $24 \%$ lower glycaemic responses than a control white bread (high-amylose bread iAUC 0-120 $\mathrm{min}=185 \pm 29.7 \mathrm{mmoL} \mathrm{L}^{-1} \mathrm{~min}^{-1}$, white control bread iAUC 0-120 $\mathrm{min}=224 \pm 27.3 \mathrm{mmoL} \mathrm{L}^{-1} \min ^{-1}$, mean \pm SEM, $n=14$ ) although all bread types in their study were baked at pumpernickel conditions (20 hours, $120^{\circ} \mathrm{C}$ ), which may have modulated starch resistance to enzymatic digestion. In the present study, the difference in starch susceptibility to amylase digestion between sbeII and WT control breads was observed using a shorter baking time (15 $\mathrm{min})$ at higher temperature $\left(185{ }^{\circ} \mathrm{C}\right)$ to produce white rolls, resembling the Chorleywood baking process.

It is noteworthy that these breads were stored at $-20{ }^{\circ} \mathrm{C}$ for $\sim 2$ months longer than those consumed by the human study participants. There is still a limited understanding of the effect of freeze-thaw treatment on starch digestibility in bread and glycaemia, however the largest effects of cold storage occur during the initial stages of freezing ${ }^{40}$ and further starch retrogradation effects thereafter would likely be minimal and negligible with regard to influencing postprandial glycaemia. ${ }^{41}$

A study by Sisson et al. in $2020^{15}$ showed that pasta made from sbeIIa mutant semolina (with 58\% amylose starch) produced pasta with lower glycaemic index (compared to an iso- glucidic WT control pasta) and acceptable quality, although the authors did report slightly reduced firmness, increased cooking loss and inferior colour compared to the control, likely to be linked to the large increase in amylose. The sbeII bread used in the present study presented minor differences in texture compared to the WT control but was deemed overall acceptable by participants.

The results presented in this study showed that the effect of bread on glycaemic response (iAUCs) were measured similarly in IF by CGM on the upper arm and in capillary blood by FP, therefore the conclusions based on iAUCs does not differ between the metrics. Measuring glucose responses in IF by CGM was found to be an effective and minimally invasive method to carry out intervention studies: sensor measurements are reliably taken every $15 \mathrm{~min}$ (this may vary depending on the type of sensor), allowing studies to collect larger glucose datasets compared to capillary glucose measured by FP, which is also more painful for study participants. Previous studies have shown a good agreement between measurements taken in blood and $\mathrm{IF}^{42}$ however, the IF measurements can vary depending on the type of device and the insertion site. ${ }^{18}$

Based on the results collected from eight participants, the effect size measured in this study in IF was consistent with the expected effect size based on in vitro amylolysis. The study was underpowered due to the COVID-19 outbreak that forced us to stop all study activities ahead of time. Nevertheless, the results from the human study suggested that bread made with sbeII mutant wheat may lead to lower postprandial IF glycaemic responses.

Overall, this study showed that the small increases in amylose content in the sbeII mutant wheat raw material can be used to produce sbeII wheat-based foods with lower starch susceptibility to hydrolysis, without substantially compromising (bread) texture or appearance, measured instrumentally. Future foods made from sbeII mutant wheat may provide an alternative to high glycaemic wheat-based staple foods, including white bread. Further acute and chronic intervention studies are needed to fully explore the potential use of sbeII mutant wheat to reduce the glycaemic potency of bread and other wheat-based foods.

\section{Abbreviations}

$\begin{array}{ll}\text { IF } & \text { Interstitial fluid } \\ \text { FP } & \text { Fingerprick } \\ \text { CGM } & \text { Continuous glucose monitoring } \\ \text { sbeII } & \text { Starch branching enzyme II } \\ \text { WT } & \text { Wild-type } \\ \text { RS } & \text { Resistant starch } \\ \text { iAUC } & \text { Incremental area under the curve }\end{array}$

\section{Authors contribution}

Marina Corrado: Conceptualization, investigation, methodology, project administration, data curation and formal ana- 
lysis, writing - original draft preparation, writing - review \& editing, Jennifer Ahn-Jarvis: investigation, methodology, writing - review \& editing, Brendan Fahy: investigation, writing - review \& editing, George M. Savva: methodology, data curation, formal analysis, Cathrina H. Edwards: conceptualization, methodology, supervision, writing - review \& editing, Brittany A. Hazard: conceptualization, funding acquisition, methodology, supervision, writing - review \& editing.

\section{Conflicts of interest}

The authors declare no conflicts of interest.

\section{Acknowledgements}

We thank Dr Natalia Perez-Moral for blinding and unblinding the intervention meals. This project was funded by the UKRI Biotechnology and Biological Sciences Research Council (BBSRC) Norwich Research Park Biosciences Doctoral Training Partnership grant number BB/M011216/1 and the BBSRC Institute Strategic Programme 'Food Innovation and Health' grant number BB/R012512/1 and its constituent projects BBS/ E/F/000PR10343 (Theme 1, Food Innovation) and BBS/E/F/ 000PR10345 (Theme 2, Digestion in the Upper GI Tract) and the BBSRC Core Capability Grant BB/CCG1860/1 awarded to Quadram Institute Bioscience, and previously by the Institute Strategic Programme Grant 'Food and Health' Grant No. BB/J004545/1 awarded to the Institute of Food Research; the BBSRC Institute Strategic Programme Grants 'Molecules from Nature' - Crop Quality BBS/E/J/000PR9799 awarded to the John Innes Centre and 'Designing Future Wheat' BB/P016855/1 awarded to the John Innes Centre.

\section{References}

1 D. M. Nathan, M. B. Davidson, R. A. DeFronzo, R. J. Heine, R. R. Henry, R. Pratley and B. Zinman, Impaired Fasting Glucose and Impaired Glucose Tolerance, Implications for Care, 2007, 30, 753-759.

2 F. de Vegt, J. M. Dekker, A. Jager, E. Hienkens, P. J. Kostense, C. D. A. Stehouwer, G. Nijpels, L. M. Bouter and R. J. Heine, Relation of Impaired Fasting and Postload Glucose With Incident Type 2 Diabetes in a Dutch PopulationThe Hoorn Study, J. Am. Med. Assoc., 2001, 285, 2109-2113.

3 C. A. Whicher, S. O'Neill and R. I. G. Holt, Diabetes in the UK: 2019, Diabetic Med., 2020, 37, 242-247.

4 Public Health England, National Diet and Nutrition Survey 2016/2017 to 2018/2019, 2020.

5 E. E. Blaak, J.-M. Antoine, D. Benton, I. Björck, L. Bozzetto, F. Brouns, M. Diamant, L. Dye, T. Hulshof, J. J. Holst, D. J. Lamport, M. Laville, C. L. Lawton, A. Meheust, A. Nilson, S. Normand, A. A. Rivellese, S. Theis, S. S. Torekov, S. Vinoy and E. Aisbl, Impact of postprandial glycaemia on health and prevention of disease, Obes. Rev., 2012, 13, 923-984.

6 T. M. S. Wolever and C. Mehling, High-carbohydrate-lowglycaemic index dietary advice improves glucose disposition index in subjects with impaired glucose tolerance, Br. J. Nutr., 2002, 87, 477-487.

7 D. J. Jenkins, C. W. Kendall, L. S. Augustin, S. Franceschi, M. Hamidi, A. Marchie, A. L. Jenkins and M. Axelsen, Glycemic index: overview of implications in health and disease, Am. J. Clin. Nutr., 2002, 76, 266s-273s.

8 C. M. McCallum, L. Comai, E. A. Greene and S. Henikoff, Targeting induced local lesions IN genomes (TILLING) for plant functional genomics, Plant Physiol., 2000, 123, 439442.

9 K. V. Krasileva, H. A. Vasquez-Gross, T. Howell, P. Bailey, F. Paraiso, L. Clissold, J. Simmonds, R. H. RamirezGonzalez, X. Wang, P. Borrill, C. Fosker, S. Ayling, A. L. Phillips, C. Uauy and J. Dubcovsky, Uncovering hidden variation in polyploid wheat, Proceedings of the National Academy of Sciences, 2017, 114, E913-E921.

10 B. Hazard, X. Zhang, P. Colasuonno, C. Uauy, D. M. Beckles and J. Dubcovsky, Induced mutations in the starch branching enzyme II (SBEII) genes increase amylose and resistant starch content in durum wheat, Crop Sci., 2012, 52, 17541766.

11 A. Schönhofen, B. Hazard, X. Zhang and J. Dubcovsky, Registration of Common Wheat Germplasm with Mutations in SBEII Genes Conferring Increased Grain Amylose and Resistant Starch Content, J. Plant Regist., 2016, 10, 200-205.

12 L. M. N. K. Ekström, I. M. E. Björck and E. M. Östman, An improved course of glycaemia after a bread based breakfast is associated with beneficial effects on acute and semiacute markers of appetite, Food Funct., 2016, 7, 1040-1047.

13 L. M. N. K. Ekström, I. M. E. Björck and E. M. Ostman, On the possibility to affect the course of glycaemia, insulinaemia, and perceived hunger/satiety to bread meals in healthy volunteers, Food Funct., 2013, 4, 495-660.

14 M. Corrado, A. Cherta-Murillo, E. S. Chambers, A. J. Wood, A. Plummer, A. Lovegrove, C. H. Edwards, G. S. Frost and B. A. Hazard, Effect of semolina pudding prepared from starch branching enzyme IIa and b mutant wheat on glycaemic response in vitro and in vivo: a randomised controlled pilot study, Food Funct., 2020, 11, 617-627.

15 M. Sissons, F. Sestili, E. Botticella, S. Masci and D. Lafiandra, Can Manipulation of Durum Wheat Amylose Content Reduce the Glycaemic Index of Spaghetti?, Foods, 2020, 9, 693.

16 D. P. Belobrajdic, A. Regina, B. Klingner, I. Zajac, S. Chapron, P. Berbezy and A. R. Bird, High-Amylose Wheat Lowers the Postprandial Glycemic Response to Bread in Healthy Adults: A Randomized Controlled Crossover Trial, J. Nutr., 2019, 149, 1335-1345.

17 S. E. Berry, A. M. Valdes, D. A. Drew, F. Asnicar, M. Mazidi, J. Wolf, J. Capdevila, G. Hadjigeorgiou, R. Davies, H. Al Khatib, C. Bonnett, S. Ganesh, E. Bakker, D. Hart, 
M. Mangino, J. Merino, I. Linenberg, P. Wyatt, J. M. Ordovas, C. D. Gardner, L. M. Delahanty, A. T. Chan, N. Segata, P. W. Franks and T. D. Spector, Human postprandial responses to food and potential for precision nutrition, Nat. Med., 2020, 26(6), 964-973.

18 R. Howard, J. Guo and K. D. Hall, Imprecision nutrition? Different simultaneous continuous glucose monitors provide discordant meal rankings for incremental postprandial glucose in subjects without diabetes, Am. J. Clin. Nutr., 2020, 112, 1114-1119.

19 L. Dye, M. Mansfield, N. Lasikiewicz, L. Mahawish, R. Schnell, D. Talbot, H. Chauhan, F. Croden and C. Lawton, Correspondence of continuous interstitial glucose measurement against arterialised and capillary glucose following an oral glucose tolerance test in healthy volunteers, Br. J. Nutr., 2010, 103, 134-140.

20 B. H. Bajka, A. M. Pinto, J. Ahn-Jarvis, P. Ryden, N. Perez-Moral, A. van der Schoot, C. Stocchi, C. Bland, S. E. Berry, P. R. Ellis and C. H. Edwards, The impact of replacing wheat flour with cellular legume powder on starch bioaccessibility, glycaemic response and bread roll quality: A double-blind randomised controlled trial in healthy participants, Food Hydrocolloids, 2021, 114, 106565.

21 C. H. Edwards, M. M. Grundy, T. Grassby, D. Vasilopoulou, G. S. Frost, P. J. Butterworth, S. E. Berry, J. Sanderson and P. R. Ellis, Manipulation of starch bioaccessibility in wheat endosperm to regulate starch digestion, postprandial glycemia, insulinemia, and gut hormone responses: a randomized controlled trial in healthy ileostomy participants, Am. J. Clin. Nutr., 2015, 102, 791-800.

22 C. H. Edwards, N. Cochetel, L. Setterfield, N. Perez-Moral and F. J. Warren, A single-enzyme system for starch digestibility screening and its relevance to understanding and predicting the glycaemic index of food products, Food Funct., 2019, 10, 4751-4760.

23 C. H. Edwards, F. J. Warren, P. J. Milligan, P. J. Butterworth and P. R. Ellis, A novel method for classifying starch digestion by modelling the amylolysis of plant foods using first order enzyme kinetic principles, Food Funct., 2014, 5, 27512758.

24 R Development Core Team, R: A language and environment for statistical computing, $R$ Foundation for Statistical Computing, 2008.

25 RStudio Team, RStudio: Integrated Development for $\mathrm{R}, R$ Foundation for Statistical Computing, 2019.

26 C. S. McCamy, H. Marcus and J. G. Davidson, A color-rendition chart, J. Appl. Photogr. Eng., 1976, 2, 95-99.

27 M. Maskan, Kinetics of colour change of kiwifruits during hot air and microwave drying, J. Food Eng., 2001, 48, 169175.

28 C. Byrne, D. Blunt, J. Burn, E. Chambers, A. Dagbasi, G. Franco Becker, G. Gibson, L. Mendoza, K. Murphy, C. Poveda, A. Ramgulam, M. Tashkova, G. Walton, C. Washirasaksiri and G. Frost, A study protocol for a randomised crossover study evaluating the effect of diets differing in carbohydrate quality on ileal content and appetite regulation in healthy humans, F1000Research, 2019, 8, 258.

29 L. A. H. Rosén, L. O. B. Silva, U. K. Andersson, C. Holm, E. M. Östman and I. M. Björck, Endosperm and whole grain rye breads are characterized by low post-prandial insulin response and a beneficial blood glucose profile, Nutr. J., 2009, 8, 1-11.

30 F. Brouns, I. Bjorck, K. N. Frayn, A. L. Gibbs, V. Lang, G. Slama and T. M. S. Wolever, Glycaemic index methodology, Nutr. Res. Rev., 2005, 18, 145-171.

31 A. Kuznetsova, P. B. Brockhoff and R. H. B. Christensen, lmerTest Package: Tests in Linear Mixed Effects Models, J. Stat. Softw., 2017, 82, 1-16.

32 R. Lenth, emmeans: Estimated Marginal Means, aka LeastSquares Means. R package version 1.4.5, 2020.

33 H. Wickham, Reshaping Data with the reshape Package, J. Stat. Softw., 2007, 21, 1-20.

34 H. Wickham, ggplot2: Elegant Graphics for Data Analysis, Springer-Verlag New York, 2016.

35 P. J. Aphalo, ggpmisc: Miscellaneous Extensions to 'goplot2', 2021.

36 C. A. Schneider, W. S. Rasband and K. W. Eliceiri, NIH Image to ImageJ: 25 years of image analysis, Nat. Methods, 2012, 9, 671-675.

37 G. H. Anderson, C. E. Cho, T. Akhavan, R. C. Mollard, B. L. Luhovyy and E. T. Finocchiaro, Relation between estimates of cornstarch digestibility by the Englyst in vitro method and glycemic response, subjective appetite, and short-term food intake in young men, Am. J. Clin. Nutr., 2010, 91, 932-939.

38 Scientific Advisory Committee on Nutrition, Dietary reference values for energy, The Stationery Office, 2011.

39 K. M. Behall and J. Hallfrisch, Plasma glucose and insulin reduction after consumption of breads varying in amylose content, Eur. J. Clin. Nutr., 2002, 56, 913-920.

40 H. Patel, P. G. Royall, S. Gaisford, G. R. Williams, C. H. Edwards, F. J. Warren, B. M. Flanagan, P. R. Ellis and P. J. Butterworth, Structural and enzyme kinetic studies of retrograded starch: Inhibition of $\alpha$-amylase and consequences for intestinal digestion of starch, Carbohydr. Polym., 2017, 164, 154-161.

41 C. H. Edwards, A. S. Veerabahu, A. J. Mason, P. J. Butterworth and P. R. Ellis, $\alpha$-Amylase action on starch in chickpea flour following hydrothermal processing and different drying, cooling and storage conditions, Carbohydr. Polym., 2021, 259, 117738.

42 N. Peled, D. Wong and S. L. Gwalani, Comparison of glucose levels in capillary blood samples obtained from a variety of body sites, Diabetes Technol. Ther., 2002, 4, 35-44; discussion 45-37.

43 E. Hallström, F. Sestili, D. Lafiandra, I. Björck and E. Östman, A novel wheat variety with elevated content of amylose increases resistant starch formation and may beneficially influence glycaemia in healthy subjects, Food Nutr. Res., 2011, 55, 1-8. 